\title{
Generalized Bilinear Differential Operators Application in a (3+1)-Dimensional Generalized Shallow Water Equation
}

\author{
Jingzhu Wu, ${ }^{1}$ Xiuzhi Xing, ${ }^{1}$ and Xianguo Geng ${ }^{2}$ \\ ${ }^{1}$ Department of Mathematics, Zhoukou Normal University, Zhoukou 466000, China \\ ${ }^{2}$ Department of Mathematics, Zhengzhou University, Zhengzhou 450052, China \\ Correspondence should be addressed to Jingzhu Wu; wujingzhu@zknu.edu.cn
}

Received 14 April 2015; Revised 26 June 2015; Accepted 29 June 2015

Academic Editor: Mahouton N. Hounkonnou

Copyright (c) 2015 Jingzhu Wu et al. This is an open access article distributed under the Creative Commons Attribution License, which permits unrestricted use, distribution, and reproduction in any medium, provided the original work is properly cited.

\begin{abstract}
The relations between $D_{p}$-operators and multidimensional binary Bell polynomials are explored and applied to construct the bilinear forms with $D_{p}$-operators of nonlinear equations directly and quickly. Exact periodic wave solution of a $(3+1)$-dimensional generalized shallow water equation is obtained with the help of the $D_{p}$-operators and a general Riemann theta function in terms of the Hirota method, which illustrate that bilinear $D_{p}$-operators can provide a method for seeking exact periodic solutions of nonlinear integrable equations. Furthermore, the asymptotic properties of the periodic wave solutions indicate that the soliton solutions can be derived from the periodic wave solutions.
\end{abstract}

\section{Introduction}

The studies of exact solutions of nonlinear partial differential equations (NPDEs) have received considerable attention in connection with the important problems that arise in scientific applications. Many powerful methods have been proposed to obtain exact solutions of (NPDEs); a series of methods have been proposed, such as Painléve test [1], Bäcklund transformation method [2,3], Darboux transformation [4], inverse scattering transformation method [5], Lie group method [6, 7], Hamiltonian method [8,9], and the Hirota method $[10,11]$.

In order to seek the periodic solutions of nonlinear evolution equations, Porubov and Parker proposed Weierstrass elliptic function expansion method [12]; Liu et al. proposed Jacobi elliptic sine function expansion methods $[13,14]$ and obtained some exact periodic solutions of some nonlinear evolution equations. They pointed out that their method can be applied to solve the nonlinear evolution equations in which the odd- and even-order derivative terms do not coexist. Zhang [15] developed Jacobi elliptic function expansion method to solve some nonlinear evolution equations in which the odd- and even-order derivative term coexist and obtained some exact periodic solutions of the equations. The bilinear method developed by Hirota have proved to be particularly powerful in obtaining the soliton solutions, quasiperiodic wave solutions, and periodic wave solutions $[16,17]$. As we all know, once the bilinear forms of nonlinear differential equations are obtained, the multisoliton solutions, the bilinear Bäcklund transformation, and Lax pairs of NPDEs can be constructed easily. It is clear that the key of Hirota direct method is finding the bilinear forms of the given differential equations by the Hirota differential $D$-operators. However, Hirota bilinear equations are special and there are many other bilinear differential equations which are not written in the Hirota bilinear form.

In fact, solving nonlinear equations (especially nonlinear partial differential equations) is very difficult, and there is no unified method. The present methods can only be applied to a certain equation or some equations. So the work of continuing to find some effective method of solving nonlinear equations is important and meaningful. Recently, Ma put forward generalized bilinear differential operators named $D_{p}$-operators in [18], which are used to create bilinear differential equations. Furthermore, different symbols are also used to furnish relations with Bell polynomials in [19] and even for trilinear equations in [20]. In this paper, we would like to explore how to construct the bilinear forms 
with $D_{p}$-operators and how to obtain the exact solutions of nonlinear equation with the help of $D_{p}$ bilinear operators method.

The paper is structured as follows. In Section 2, we will give a brief introduction about the bilinear $D_{p}$-operators. In Section 3, we explore the relations between multivariate binary Bell polynomials and the $D_{p}$-operators. The $D_{p}$ bilinear forms of some nonlinear evolutions are given quickly and easily from the relations. In Section 4, we will use the relation in Section 2 to seek the bilinear form with $D_{p^{-}}$ operators of the $(3+1)$-dimensional generalized shallow water equation and then take advantage of the $D_{p}$-operators and the Riemann theta function $[21,22]$ to obtain its exact periodic wave solution which can be reduced to the soliton solution via asymptotic analysis.

\section{Bilinear $D_{p}$-Operators}

It is known to us that Hirota bilinear $D$-operators play a significant role in Hirota direct method. The $D$-operators are defined as follows:

$$
\begin{aligned}
D_{x}^{m} D_{y}^{k} f \cdot g= & \left(\frac{\partial}{\partial x_{1}}-\frac{\partial}{\partial x_{2}}\right)^{m}\left(\frac{\partial}{\partial y_{1}}-\frac{\partial}{\partial y_{2}}\right)^{k} \\
& \cdot\left[f\left(x_{1}, y_{1}\right) g\left(x_{2}, y_{2}\right)\right]
\end{aligned}
$$

where the right-hand side is computed in

$$
\begin{aligned}
& x_{1}=x_{2}=x, \\
& y_{1}=y_{2}=y .
\end{aligned}
$$

According to the definition of Hirota bilinear D-operators, we have

$$
\begin{aligned}
D_{x} f \cdot g & =f_{x} g-f g_{x}, \\
D_{x}^{2} f \cdot g & =f_{x x} g-2 f_{x} g_{x}+f g_{x x}, \\
D_{x} D_{t} f \cdot g= & f_{x t} g-f_{x} g_{t}-f_{t} g_{x}+f g_{x t}, \\
D_{x}^{3} f \cdot g= & f_{x x x} g-3 f_{x x} g_{x}+3 f_{x} g_{x x}-f g_{x x x}, \\
D_{t} D_{x}^{3} f \cdot g= & f_{3 x t} g-f_{3 x} g_{t}-3 f_{2 x t} g_{x}+3 f_{2 x} g_{x t} \\
& +3 f_{x t} g_{2 x}-3 f_{x} g_{2 x t}-f_{t} g_{3 x}+f g_{3 x t} .
\end{aligned}
$$

Based on the Hirota D-operators, Professor Ma put forward a kind of bilinear $D_{p}$-operators in [18]:

$$
\begin{aligned}
& D_{p, x}^{m} D_{p, y}^{k}\left[f\left(x_{1}, y_{1}\right) g\left(x_{2}, y_{2}\right)\right] \\
& \quad=\left.\left(\frac{\partial}{\partial x_{1}}+\alpha \frac{\partial}{\partial x_{2}}\right)^{m}\left(\frac{\partial}{\partial y_{1}}+\alpha \frac{\partial}{\partial y_{2}}\right)^{k}\right|_{x_{1}=x_{2}=x, y_{1}=y_{2}=y},
\end{aligned}
$$

where the powers of $\alpha$ are determined by

$$
\alpha^{i}=(-1)^{r(i)}
$$

where $i=r(i) \bmod p$ with $0 \leq r(i)<p ; i \geq 0$.
Obviously, the case of $p=1$ gives the normal derivatives, and the cases of $p=2 k, k \in N$, reduce to Hirota bilinear operators.

In particular, when $m=0$, we have

$$
\begin{aligned}
D_{p, x}^{n}(f \cdot g)(x) & =\left.\left(\partial_{x}+\alpha \partial_{x^{\prime}}\right)^{n} f(x) g\left(x^{\prime}\right)\right|_{x^{\prime}=x} \\
& =\sum_{i=0}^{n} \alpha^{i} C_{n}^{i} \partial_{x}^{n-i} f \partial_{x}^{i} g .
\end{aligned}
$$

According to the definition of $D_{p}$-operator, when $p=3$, we have

$$
\begin{aligned}
\alpha^{0}= & 1, \\
\alpha & =-1, \\
\alpha^{2}= & \alpha^{3}=1, \\
\alpha^{4}= & -1, \\
\alpha^{5}= & \alpha^{6}=1, \\
\alpha^{7}= & -1, \\
\alpha^{8}= & \alpha^{9}=1, \ldots, \\
D_{3, x}^{4} f \cdot g= & \sum_{i=0}^{4} \alpha^{i} C_{4}^{i} \partial_{x}^{4-i} f \partial_{x}^{i} g \\
= & f_{4 x} g-4 f_{3 x} g_{x}+6 f_{2 x} g_{2 x}+4 f_{x} g_{3 x} \\
& -f g_{4 x} ;
\end{aligned}
$$

when $p=5$, we have

$$
\begin{aligned}
\alpha^{0} & =1, \\
\alpha^{1} & =-1, \\
\alpha^{2} & =1, \\
\alpha^{3} & =-1, \\
\alpha^{4} & =\alpha^{5}=1, \\
\alpha^{6} & =-1, \\
\alpha^{7} & =1, \\
\alpha^{8} & =-1, \ldots, \\
D_{5, x} f \cdot g & =f_{x} g-f g_{x}, D_{5, t} D_{5, x} f \cdot g \\
& =D_{5, t}\left(f_{x} g-f g_{x}\right) \\
& =f_{x t} g-f_{x} g_{t}-f_{t} g_{x}+f g_{x t}, \\
D_{5, x}^{2} f \cdot g & \sum_{i=0}^{2} \alpha^{i} C_{2}^{i} \partial_{x}^{2-i} f \partial_{x}^{i} g=f_{x x} g-2 f_{x} g_{x}+f g_{x x},
\end{aligned}
$$




$$
\begin{aligned}
D_{5, x}^{4} f \cdot g= & \sum_{i=0}^{4} \alpha^{i} C_{4}^{i} \partial_{x}^{4-i} f \partial_{x}^{i} g \\
= & f_{4 x} g-4 f_{3 x} g_{x}+6 f_{x x} g_{x x}-4 f_{x} g_{x x} \\
& +f g_{4 x}, \\
D_{5 x}^{5} f \cdot g= & \sum_{i=0}^{5} \alpha^{i} C_{5}^{i} \partial_{x}^{5-i} f \partial_{x}^{i} g \\
= & f_{5 x} g-5 f_{4 x} g_{x}+10 f_{3 x} g_{2 x}-10 f_{2 x} g_{3 x} \\
& +5 f_{x} g_{4 x}+f g_{5 x} .
\end{aligned}
$$

Now, under $u=2(\ln f)_{x x}$, for Kdv equation,

$$
u_{t}+6 u u_{x}+u_{x x x}=0
$$

we have

$$
\frac{\partial}{\partial x}\left[\frac{\left(f_{x t} f-f_{x} f_{t}+f_{4 x} f-4 f_{3 x} f_{x}+3 f_{2 x}^{2}\right)}{f^{2}}\right]=0 ;
$$

we can get its bilinear form with $D_{p}$-operators:

$$
\left(D_{5, x} D_{5, t}+D_{5 x}^{4}\right) f \cdot f=0 .
$$

In fact, if we seek the bilinear form with $D_{p}$-operators of nonlinear integrable differential equations according to the definition of $D_{p}$-operators, this needs some special skills and complex computations. So we would like to explore the relations between $D_{p}$-operators and multivariate binary Bell polynomials. The bilinear forms with $D_{p}$-operators of nonlinear integrable differential equations are obtained quickly and easily by applying the relations.

\section{Relations with Bell Exponential Polynomials}

3.1. Relations with Bell Exponential Polynomials. As we all know, Bell proposed three kinds of exponent form polynomials. Later, Wang and Chen generalized the third type of Bell polynomials in $[23,24]$ which is used mainly in this paper. The multidimensional binary Bell polynomials which we will use are defined as follows:

$$
\begin{aligned}
Y_{n_{1} x_{1}, \ldots, n_{l} x_{l}}(y) & =Y_{n_{1}, \ldots, n_{l}}\left(y_{r_{1} x_{1}}, \ldots, y_{r_{l} x_{l}}\right) \\
& =e^{-y} \partial_{x_{1}}^{n_{1}} \ldots \partial_{x_{l}}^{n_{l}} e^{y} \quad\left(n_{1}, \ldots, n_{l} \geq 0\right),
\end{aligned}
$$

with $y_{r_{1} x_{1}, \ldots, r_{l} x_{l}}=\partial_{x_{1}}^{n_{1}} \ldots \partial_{x_{l}}^{n_{l}}, r_{1}=0, \ldots, n_{1}, \ldots, r_{l}=0, \ldots, n_{l}$.
For example,

$$
\begin{aligned}
& Y_{x}=y_{x} \\
& Y_{2 x}=y_{x}^{2}+y_{2 x}, \\
& Y_{3 x}=y_{x}^{3}+3 y_{x} y_{2 x}+y_{3 x}, \\
& Y_{4 x}=y_{x}^{4}+4 y_{x} y_{3 x}+6 y_{x}^{2} y_{2 x}+3 y_{2 x}^{2}+y_{4 x}, \\
& Y_{5 x}=y_{x}^{5}+5 y_{x} y_{4 x}+15 y_{x} y_{2 x}^{2}+10 y_{x}^{2} y_{3 x}+10 y_{2 x} y_{3 x} \\
& \quad+10 y_{x}^{3} y_{x x}+y_{5 x} \\
& Y_{6 x}=\left(y_{x}^{5}+5 y_{x} y_{4 x}+15 y_{x} y_{2 x}^{2}+10 y_{x}^{2} y_{3 x}+10 y_{2 x} y_{3 x}\right. \\
& \left.\quad+10 y_{x}^{3} y_{x x}+y_{5 x}\right)_{x}+\left(y_{x}^{5}+5 y_{x} y_{4 x}+15 y_{x} y_{2 x}^{2}\right. \\
& \left.\quad+10 y_{x}^{2} y_{3 x}+10 y_{2 x} y_{3 x}+10 y_{x}^{3} y_{x x}+y_{5 x}\right)_{x} y_{x} ; \\
& Y_{x, t}=y_{x}^{2}+y_{x t}, \\
& Y_{2 x, t}=2 y_{x} y_{x t}+y_{2 x, t}+y_{x}^{2} y_{t}+y_{2 x} y_{t}, \\
& Y_{3 x, t}=3 y_{x}^{2} y_{t}+3 y_{x t} y_{2 x}+3 y_{x} y_{2 x, t}+y_{3 x, t}+\left(y_{x}^{3}\right. \\
& \left.\quad+3 y_{x} y_{2 x}+y_{3 x}\right) y_{t} .
\end{aligned}
$$

For the sake of computational convenience, we assume that

$$
\begin{aligned}
& f=e^{\xi\left(x_{1}, \ldots, x_{l}\right)}, \\
& g=e^{\eta\left(x_{1}, \ldots, x_{l}\right)} ;
\end{aligned}
$$

we have

$$
\begin{aligned}
(f g)^{-1} D_{p, x_{1}}^{n_{1}}, \ldots, D_{p, x_{l}}^{n_{l}} f \cdot g & \\
= & \sum_{k_{1}=0}^{n_{1}} \ldots \sum_{k_{l}=0}^{n_{1}} \prod_{i=1}^{l} \alpha^{k_{i}}\left(\begin{array}{c}
n_{i} \\
k_{i}
\end{array}\right)\left(e^{-\xi} \partial_{x_{1}}^{n_{1}-k_{1}} \ldots \partial_{x_{l}}^{n_{l}-k_{l}} e^{\xi}\right) \\
\cdot & \left(e^{-\eta} \partial_{x_{1}}^{n_{1}-k_{1}} \cdots \partial_{x_{l}}^{n_{l}-k_{l}} e^{\eta}\right) \\
= & \sum_{k_{1}=0}^{n_{1}} \ldots \sum_{k_{l}=0}^{n_{1}} \prod_{i=1}^{l} \alpha^{k_{i}}\left(\begin{array}{c}
n_{i} \\
k_{i}
\end{array}\right) Y_{\left(n_{1}-k_{1}\right) x_{1}, \ldots,\left(n_{l}-k_{l}\right) x_{l}}(\xi) \\
\cdot & Y_{k_{1} x_{1}, \ldots, k_{l} x_{l}}(\eta)=\sum_{k_{1}=0}^{n_{1}} \ldots \sum_{k_{l}=0}^{n_{1}} \prod_{i=1}^{l} \alpha^{k_{i}}\left(\begin{array}{c}
n_{i} \\
k_{i}
\end{array}\right) \\
\cdot & Y_{\left(n_{1}-k_{1}\right) x_{1}, \ldots,\left(n_{l}-k_{l}\right) x_{l}}\left(\xi_{r_{1}, \ldots, r_{l}}\right) \\
\cdot & Y_{k_{1} x_{1}, \ldots, k_{l} x_{l}}\left(\alpha^{r_{1}+\cdots+r_{l}} \eta_{r_{1}, \ldots, r_{l}}\right)=Y_{n_{1}, \ldots, n_{l}}\left(y_{r_{1}, \ldots, r_{l}}\right. \\
= & \left.\xi_{r_{1}, \ldots, r_{l}}+\alpha^{r_{1}+\cdots+r_{l}} \eta_{r_{1}, \ldots, r_{l}}\right)=\bar{Y}_{p ; n_{1} x_{1}, \ldots, n_{l} x_{l}}(v, w),
\end{aligned}
$$

where

$$
\begin{gathered}
w=\xi+\eta, \\
v=\xi-\eta .
\end{gathered}
$$


We find that the link between $\bar{Y}$-polynomials and the $D_{p}$ operator can be given in the following through the above deduction:

$$
\begin{aligned}
& (f g)^{-1} D_{p, x_{1}}^{n_{1}}, \ldots, D_{p, x_{l}}^{n_{l}} f \cdot g \\
& \quad=\bar{Y}_{p ; n_{1} x_{1}, \ldots, n_{l} x_{l}}\left(v=\ln \frac{f}{g}, w=\ln f g\right) \\
& \quad=Y_{n_{1}, \ldots, n_{l}}\left(y_{r_{1}, \ldots, r_{l}}=\xi_{r_{1}, \ldots, r_{l}}+\alpha^{r_{1}+\cdots+r_{l}} \eta_{r_{1}, \ldots, r_{l}}\right) .
\end{aligned}
$$

In particular, when $f=g$, (17) becomes

$$
\begin{gathered}
f^{-2} D_{p, x_{1}}^{n_{1}}, \ldots, D_{p, x_{l}}^{n_{l}} f \cdot f=\bar{P}_{p ; n_{1} x_{1}, \ldots, n_{l} x_{l}}(q) \\
=\bar{Y}_{p ; n_{1} x_{1}, \ldots, n_{l} x_{l}}(v=0, w=2 \ln f=q) .
\end{gathered}
$$

Equation (18) give the relations between $D_{p}$-operators and multivariate binary Bell polynomials.

Then we have

$$
\begin{aligned}
& D_{p, x_{1}}^{n_{1}}, \ldots, D_{p, x_{l}}^{n_{l}} f \cdot f=f^{2} \bar{P}_{p ; n_{1} x_{1}, \ldots, n_{l} x_{l}}(q) \\
& \quad=f^{2} \bar{Y}_{p ; n_{1} x_{1}, \ldots, n_{l} x_{l}}(v=0, w=2 \ln f=q) .
\end{aligned}
$$

From (13) and (18), we have

$$
\begin{aligned}
\bar{P}_{3 ; 2 x} & =\bar{P}_{5 ; 2 x}=\bar{P}_{7 ; 2 x}=q_{x x}, \\
\bar{P}_{3 ; 4 x} & =3 q_{2 x}^{2}, \\
\bar{P}_{3 ; x, t} & =\bar{P}_{5 ; x, t}=\bar{P}_{7 ; x, t}=q_{x t}, \\
\bar{P}_{5 ; 4 x} & =\bar{P}_{7 ; 4 x}=q_{4 x}+3 q_{2 x}^{2}, \\
\bar{P}_{5 ; 3 x, y} & =\bar{P}_{7 ; 3 x, y}=q_{3 x, y}+3 q_{x x} q_{x y}, \\
\bar{P}_{5 ; 2 x, t} & =0, \\
\bar{P}_{5 ; 6 x} & =15 q_{2 x}^{3}+15 q_{2 x} q_{4 x}, \\
\bar{P}_{7 ; 6 x} & =15 q_{2 x}^{3}+15 q_{2 x} q_{4 x}+q_{6 x} .
\end{aligned}
$$

3.2. Bilinear Form with $D_{p}$-Operators. In this section, we will construct the bilinear forms for $\mathrm{Kdv}$ equation, $(2+1)$ dimensional Kdv equation, and (2+1)-dimensional SawadaKotera equation with the $D_{p}$-operators quickly and easily by utilizing the relations between $D_{p}$-operators and multidimensional bilinear Bell polynomials.

Example 1 (Kdv equation). Consider

$$
u_{t}+6 u u_{x}+u_{x x x}=0
$$

Setting $u=q_{2 x}$, substituting it into (21), and integrating with respect to $x$ yield

$$
q_{x t}+3 q_{2 x}^{2}+q_{4 x}-\lambda_{1}=0
$$

where $\lambda_{1}$ is an arbitrary function of $t$.
Based on (20) and (22), (21) can be written as follows:

$$
\bar{P}_{5, x, t}(q)+\bar{P}_{5,4 x}(q)-\lambda_{1}=0 .
$$

From (19) and (23), we get the bilinear form with $D_{p^{-}}$ operators of (21)

$$
\left(D_{5, x} D_{5, t}+D_{5, x}^{4}\right) f \cdot f-\lambda_{1} f^{2}=0 .
$$

Example 2 ((2+1)-dimensional Kdv equation). Consider

$$
u_{t}+3 u u_{y}+u_{x x y}+3 u_{x} \int u_{y} d x=0
$$

Setting $u=q_{2 x}$, substituting it into (25), and integrating with respect to $x$ yield

$$
q_{x t}+3 q_{x y} q_{2 x}^{2}+q_{3 x, y}-\lambda_{2}=0,
$$

where $\lambda_{2}$ is an arbitrary function of $y, t$. Based on (20) and (26), (25) can be written as follows:

$$
\bar{P}_{5, x, t}(q)+\bar{P}_{5,3 x, y}(q)-\lambda_{2}=0 .
$$

From (19) and (27), we get the bilinear form with $D_{p^{-}}$ operators of (25):

$$
\left(D_{5, x} D_{5, t}+D_{5, x}^{3} D_{5, y}\right) f \cdot f-\lambda_{2} f^{2}=0 .
$$

Example 3 ((2+1)-dimensional Sawada-Kotera equation). Consider

$$
\begin{gathered}
u_{t}-\left(u_{4 x}+5 u u_{2 x}+\frac{5}{3} u^{3}+5 u_{x y}\right)_{x}+5 \int u_{2 y} d x \\
-5 u u_{y}-5 u_{x} \int u_{y} d x=0 .
\end{gathered}
$$

Setting $u=3 q_{2 x}$, substituting it into (29), and integrating with respect to $x$ yield

$$
\begin{aligned}
q_{x t}+ & 5 q_{2 y}-\left(q_{6 x}+15 q_{2 x} q_{4 x}+15 q_{2 x}^{3}\right) \\
& -5\left(q_{3 x y}+3 q_{2 x} q_{x y}\right)-\lambda_{3}=0
\end{aligned}
$$

where $\lambda_{3}$ is an arbitrary function of $y, t$. Based on (20) and (30), (29) can be written as follows:

$$
\begin{aligned}
& \bar{P}_{7, x, t}(q)+5 \bar{P}_{7,2 y}(q)-5 \bar{P}_{7,3 x, y}(q)-\bar{P}_{7,6 x}(q)-\lambda_{3} \\
& \quad=0 .
\end{aligned}
$$

From (19) and (31), we get the bilinear form with $D_{p^{-}}$ operators of (29):

$$
\begin{aligned}
& \left(D_{7, x} D_{7, t}+5 D_{7, y}^{2}-5 D_{7, x}^{3} D_{7, y}-D_{7, x}^{6}\right) f \cdot f-\lambda_{3} f^{2} \\
& \quad=0 .
\end{aligned}
$$

From the above computation process for seeking the bilinear forms of three nonlinear equation, we can find that the bilinear forms with $D_{p}$-operators of nonlinear integrable differential equations are obtained quickly and easily by appling the relations between $D_{p}$-operators and multidimensional bilinear Bell polynomials. 


\section{Periodic Wave Solution of the $(3+1)$-Dimensional Generalized Shallow Water Equation}

In this section, firstly, we will give the bilinear form of a $(3+1)$-dimensional generalized shallow water equation with the help of $\bar{P}$-polynomials and the $D_{p}$-operators. And then, we construct the exact periodic wave solution of the $(3+1)$ dimensional generalized shallow water equation with the aid of the Riemann theta function, $D_{p}$-operators, and the special property of the $D_{p}$-operators when acting on exponential functions.

The following is $(3+1)$-dimensional generalized shallow water equation:

$$
u_{x x x y}+3 u_{x x} u_{y}+3 u_{x} u_{x y}-u_{y t}-u_{x z}=0 .
$$

Setting $u=q_{x}$, inserting it into (33), and integrating with respect to $x$ yield

$$
q_{3 x, y}+3 q_{2 x} q_{x, y}-q_{y, t}-q_{x, z}-\lambda=0,
$$

where $\lambda$ is an arbitrary function of $y, z, t$. Based on (20) and (34), (33) can be expressed as

$$
-\bar{P}_{5 ; y, t}-\bar{P}_{5 ; x, z}+\bar{P}_{5 ; 3 x, y}-\lambda=0 .
$$

From the above, we can get the bilinear form of (33):

$$
\left(-D_{5 ; y} D_{5 ; t}-D_{5 ; x} D_{5 ; z}+D_{5 ; x}^{3} D_{5 ; y}\right) f \cdot f-\lambda \cdot f^{2}=0
$$

with $q=2 \ln f$. When acting on exponential functions, we find that $D_{p}$-operators have a good property:

$$
\begin{aligned}
G & \left(D_{p, x_{1}}, \ldots, D_{p, x_{l}}\right) e^{\xi_{1}} \cdot e^{\xi_{2}} \\
& =G\left(k_{1}+\alpha k_{2}, l_{1}+\alpha l_{2}, h_{1}+\alpha h_{2}, \omega_{1}+\alpha \omega_{2}\right) e^{\xi_{1}+\xi_{2}}, \\
\xi_{i} & =k_{i} x+l_{i} y+h_{i} z+\omega_{i} t+\xi_{i}^{(0)}, \quad i=1,2, \ldots
\end{aligned}
$$

In order to construct periodic wave solutions of (33), we study the multidimensional Riemann theta function with genus $N$ given by

$$
f(\xi)=f(\xi, \tau)=\sum_{n \in z^{N}} e^{-\pi i\langle\tau n, n\rangle+2 \pi i\langle\xi, n\rangle}
$$

in which $n=\left(n_{1}, n_{2}, \ldots, n_{N}\right)^{T} \in z^{N}$ denotes the integer value vector and $\xi=\left(\xi_{1}, \xi_{2}, \ldots, \xi_{N}\right)$ is complex phase variable. In addition, for the given two vectors $h=\left(h_{1}, h_{2}, \ldots, h_{N}\right)$ and $g=\left(g_{1}, g_{2}, \ldots, g_{N}\right)$ their inner product can be written by

$$
\langle h, g\rangle=h_{1} g_{1}+h_{2} g_{2}+\cdots+h_{N} g_{N}
$$

$-i \tau=\left(-i \tau_{i j}\right)$ in (39) is a positive definite and real-valued symmetric $N \times N$ matrix, which can be called the period matrix of the theta function. The entries $\tau_{i j}$ of $\tau$ are free parameters of the theta function (39); we consider that Riemann's (39) converges to a real-valued function with an arbitrary vector $\xi \in C^{N}$.

In what follows we construct the one-periodic wave solutions of (33). For $N=1$, Riemann theta function (39) reduces Fourier series in $n$ as follows:

$$
f=\sum_{n=-\infty}^{+\infty} e^{\pi i n^{2} \tau+2 \pi i n \eta}
$$

where $n \in Z, \tau \in C, \operatorname{Im} \tau>0$, and $\eta=k x+l y+h z+\omega t$, with $k, l, h$, and $\omega$ being constants to be determined.

Riemann theta function (41) satisfying the bilinear equation (36) yields the sufficient conditions for obtaining periodic waves. Substituting the theta function (41) into the left of (36) and using the property (37), we have

$$
\begin{aligned}
G & \left(D_{p, x}, D_{p, y}, D_{p, z}, D_{p, t}\right) f \cdot f=\sum_{n=-\infty}^{+\infty} \sum_{m=-\infty}^{+\infty} G\left(D_{p, x}, D_{p, y}, D_{p, z}, D_{p, t}\right) e^{2 \pi i n \eta+\pi i n^{2} \tau} e^{2 \pi i m \eta+\pi i m^{2} \tau} \\
& =\sum_{n=-\infty}^{+\infty} \sum_{m=-\infty}^{+\infty} G(2 \pi i(n+\alpha m) k, 2 \pi i(n+\alpha m) l, 2 \pi i(n+\alpha m) h, 2 \pi i(n+\alpha m) \omega) e^{2 \pi i(n+m) \eta+\pi i\left(n^{2}+m^{2}\right) \tau} \\
& =\sum_{\delta=-\infty}^{+\infty}\left\{\sum_{n=-\infty}^{+\infty} G\left(\left(2 \pi i\left((1-\alpha) n-\alpha^{2} \delta\right)\right) k,\left(2 \pi i\left((1-\alpha) n-\alpha^{2} \delta\right)\right) l,\left(2 \pi i\left((1-\alpha) n-\alpha^{2} \delta\right)\right) h,\left(2 \pi i\left((1-\alpha) n-\alpha^{2} \delta\right)\right) \omega\right)\right. \\
& \left.\cdot e^{\pi i\left(n^{2}+(n+\alpha \delta)^{2}\right) \tau}\right\} e^{2 \pi i(-\alpha \delta) \eta}=\sum_{\delta=-\infty}^{+\infty} \bar{G}(\delta) e^{2 \pi i(-\alpha \delta) \eta},
\end{aligned}
$$

where $\delta=-(1 / \alpha)(m+n)$. To the bilinear form of $(33), \bar{G}(\delta)$ satisfies the period characters when $p=5$. The powers of $\alpha$ obey rule (5), noting that

$$
\begin{gathered}
\bar{G}(\delta)=\sum_{n=-\infty}^{+\infty} G\left(\left(2 \pi i\left((1-\alpha) n-\alpha^{2} \delta\right)\right)\right. \\
\cdot k,\left(2 \pi i\left((1-\alpha) n-\alpha^{2} \delta\right)\right)
\end{gathered}
$$




$$
\begin{aligned}
& \cdot l,\left(2 \pi i\left((1-\alpha) n-\alpha^{2} \delta\right)\right) \\
& \left.\cdot h,\left(2 \pi i\left((1-\alpha) n-\alpha^{2} \delta\right)\right) \omega\right) e^{\pi i\left(n^{2}+(n+\alpha \delta)^{2}\right) \tau} \\
& =\sum_{n=-\infty}^{+\infty} G(2 \pi i(2 n-\delta) k, 2 \pi i(2 n-\delta) \\
& \cdot l, 2 \pi i(2 n-\delta) h, 2 \pi i(2 n-\delta) \omega) e^{\pi i\left(n^{2}+(\delta-n)^{2}\right) \tau} \\
& =\sum_{n=-\infty}^{+\infty} G(2 \pi i(2 s-(\delta-2)) k, 2 \pi i(2 s-(\delta-2)) \\
& \cdot l, 2 \pi i(2 s-(\delta-2)) h, 2 \pi i(2 s-(\delta-2)) \omega) \\
& \cdot e^{\pi i\left(s^{2}+(\delta-s-2)^{2}\right) \tau} e^{2 \pi i(\delta-1) \tau}=\bar{G}(\delta-2) e^{2 \pi i(\delta-1) \tau},
\end{aligned}
$$

where $s=n+\alpha$. From (43) we can infer that

$$
\begin{aligned}
& \bar{G}(\delta)= \begin{cases}\bar{G}(0) e^{\pi i n \delta \tau}, & \delta=2 n ; \\
\bar{G}(1) e^{\pi i\left(2 n+2 n^{2}\right)(\delta+1) \tau}, \quad \delta=2 n+1,\end{cases} \\
& \bar{G}(0)=\sum_{n=-\infty}^{\infty}\left\{-[2 \pi i(1-\alpha) n]^{2} l \omega\right. \\
& -[2 \pi i(1-\alpha) n]^{2} k h \\
& \left.+[2 \pi i(1-\alpha) n k]^{3} 2 \pi i(1-\alpha) n l-\lambda\right\} e^{2 \pi i n^{2} \tau} \\
& =\sum_{n=-\infty}^{\infty}\left(16 \pi^{2} n^{2} l \omega+16 \pi^{2} n^{2} k h+256 \pi^{4} n^{4} k^{3} l-\lambda\right) \\
& \cdot e^{2 \pi i n^{2} \tau}=0, \\
& \bar{G}(1)=\sum_{n=-\infty}^{\infty}\left\{-2 \pi i\left((1-\alpha) n-\alpha^{2}\right) l\right. \\
& +2 \pi i\left((1-\alpha) n-\alpha^{2}\right) \omega \\
& +2 \pi i\left((1-\alpha) n-\alpha^{2}\right) k 2 \pi i\left((1-\alpha) n-\alpha^{2}\right) h \\
& \left.+2 \pi i\left((1-\alpha) n-\alpha^{2}\right) k\right]^{3} 2 \pi i\left((1-\alpha) n-\alpha^{2}\right) l \\
& +2 \pi i\left(n^{2}-2 n+1\right) \tau=\sum_{n=-\infty}^{\infty}\left[4 \pi^{2}(2 n-1)^{2} l \omega\right.
\end{aligned}
$$

Also, the powers of $\alpha$ obey rule (5). For the sake of computational convenience, we denote that

$$
\begin{aligned}
& \rho_{1}(n)=e^{2 \pi i n^{2} \tau}, \\
& a_{11}=\sum_{n=-\infty}^{\infty} 16 \pi^{2} \ln ^{2} \rho_{1}(n),
\end{aligned}
$$

$$
\begin{aligned}
& a_{12}=\sum_{n=-\infty}^{\infty} \rho_{1}(n), \\
& b_{1}=-\sum_{n=-\infty}^{\infty}\left\{16 \pi^{2} n^{2} k h+256 \pi^{4} n^{4} k^{3} l\right\} \rho_{1}(n), \\
& \rho_{2}(n)=e^{\pi i\left(2 n^{2}-2 n+1\right) \tau}, \\
& a_{21}=\sum_{n=-\infty}^{\infty} n^{2} 4 \pi^{2}(2 n-1)^{2} l \rho_{2}(n), \\
& a_{22}=\sum_{n=-\infty}^{\infty} \rho_{2}(n), \\
& b_{2}=-\sum_{n=-\infty}^{\infty}\left(4 \pi^{2}(2 n-1)^{2} n^{2} k h+16 \pi^{4}(2 n-1)^{4} k^{3} l\right) \\
& \cdot \rho_{2}(n) .
\end{aligned}
$$

Then (45) and (46) can be written as

$$
\begin{aligned}
& a_{11} \omega+a_{12} \lambda-b_{1}=0, \\
& a_{21} \omega+a_{22} \lambda-b_{2}=0 .
\end{aligned}
$$

Solving this system, we get

$$
\begin{aligned}
& \omega=\frac{b_{1} a_{22}-b_{2} a_{12}}{a_{11} a_{22}-a_{12} a_{21}}, \\
& \lambda=\frac{b_{2} a_{11}-b_{1} a_{21}}{a_{11} a_{22}-a_{12} a_{21}} .
\end{aligned}
$$

Finally, we get one-periodic wave solution:

$$
u=2(\ln f)_{x},
$$

where $f$ is given by (41) and $\omega, \lambda$ are satisfied with (49). And if we assume that $k=0.01, l=0.01, h=0.01$, and $\tau=i$ to (50), the solution (50) of (33) can be shown in Figure 1.

To this end, the soliton solution of (33) can be obtained when we consider limit of the periodic solution (50). Then, assuming $e^{\pi i \tau}=\gamma$, we can obtain that

$$
\begin{aligned}
a_{11} & =\sum_{n=-\infty}^{\infty} 16 \pi^{2} \ln ^{2} e^{2 \pi i n^{2} \tau}=32 \pi^{2} l\left(\gamma^{2}+4 \gamma^{8}+\cdots\right), \\
a_{12} & =\sum_{n=-\infty}^{\infty} e^{2 \pi i n^{2} \tau}=1+2 \gamma^{2}+2 \gamma^{8}+2 \gamma^{18}+\cdots, \\
a_{21} & =\sum_{n=-\infty}^{\infty} n^{2} 4 \pi^{2}(2 n-1)^{2} l e^{\pi i\left(2 n^{2}-2 n+1\right) \tau}=8 \pi^{2} l(\gamma \\
& \left.+9 \gamma^{5}+\cdots\right), \\
a_{22} & =\sum_{n=-\infty}^{\infty} e^{\pi i\left(2 n^{2}-2 n+1\right) \tau}=\gamma+\gamma^{5}+\cdots,
\end{aligned}
$$




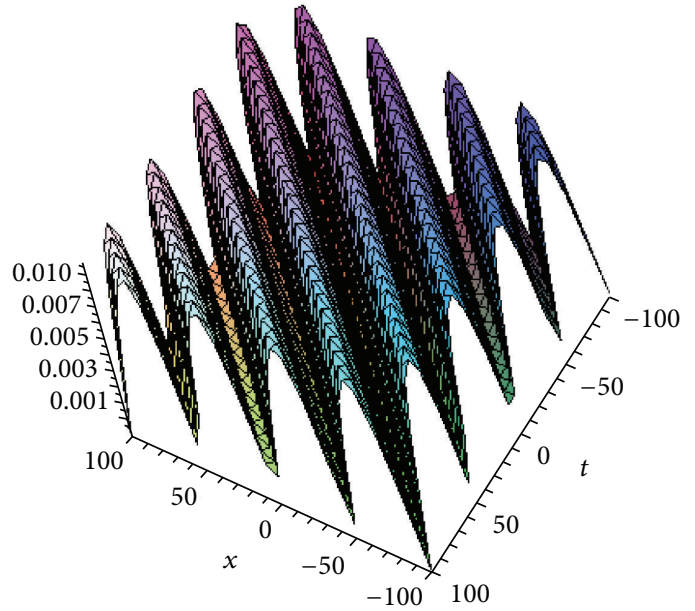

(a)

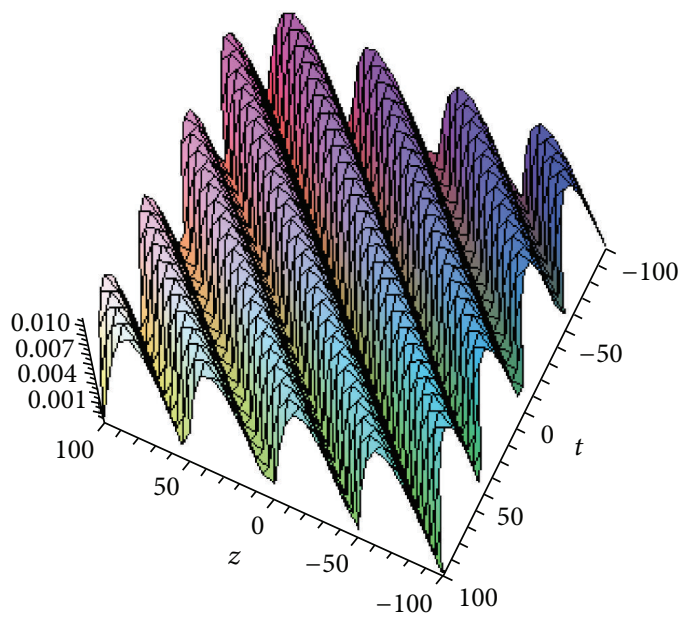

(c)

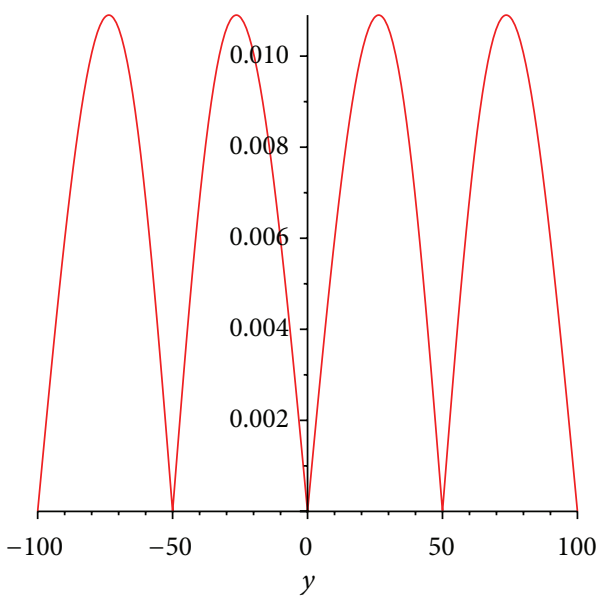

(e)

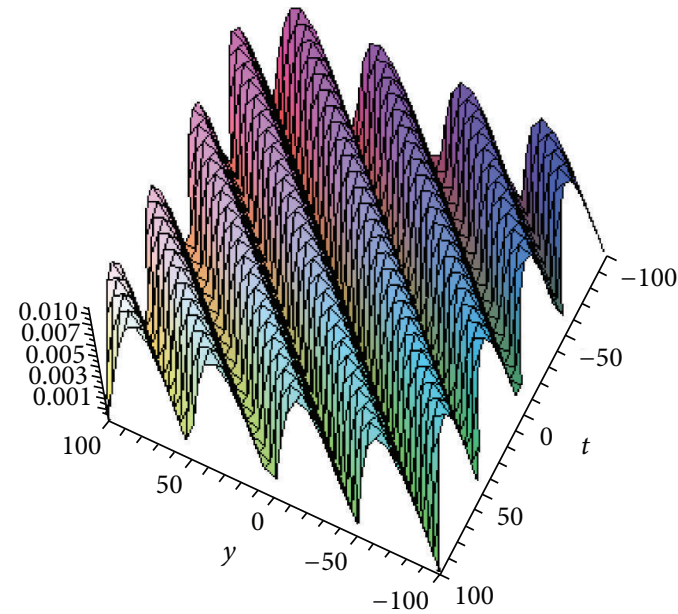

(b)

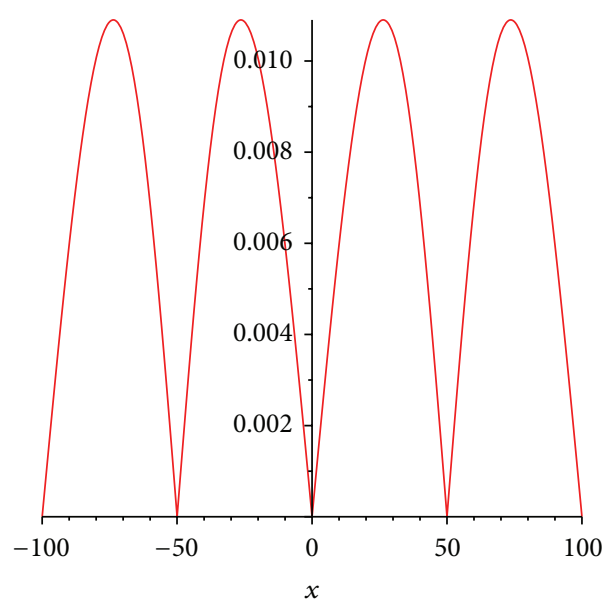

(d)

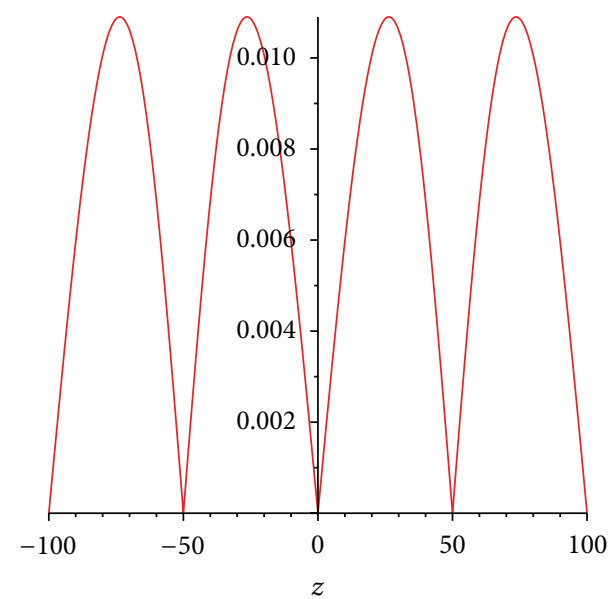

(f)

FIGURE 1: A one-periodic wave (50) of the (3+1)-dimensional shallow water wave equation (33) with parameters $k=0.01, l=0.01, h=0.01$, and $\tau=i$. This figure shows that every one-periodic wave is one-dimensional, and it can be viewed as a superposition of overlapping solitary waves, placed one period apart. (a) Perspective view of the periodic wave Abs $(u)$ on $x$ ot-plane. (b) Perspective view of the periodic wave $\operatorname{Abs}(u)$ on $y o t$-plane. (c) Perspective view of the periodic wave Abs $(u)$ on $z$ ot-plane. (d) Wave propagation pattern of the wave along the $x$-axis. (e) Wave propagation pattern of the wave along the $y$-axis. (f) Wave propagation pattern of the wave along the $z$-axis. 


$$
\begin{aligned}
b_{1}= & -\sum_{n=-\infty}^{\infty}\left\{16 \pi^{2} n^{2} k h+256 \pi^{4} n^{4} k^{3} l\right\} e^{2 \pi i n^{2} \tau} \\
& =-32 \pi^{2} k\left[\left(h+16 \pi^{2} k^{2} l\right) \gamma^{2}+4\left(h+16 \pi^{2} k^{2} l\right) \gamma^{8}\right. \\
& +\cdots], \\
b_{2}= & -\sum_{n=-\infty}^{\infty}\left(4 \pi^{2}(2 n-1)^{2} n^{2} k h+16 \pi^{2}(2 n-1)^{4} k^{3} l\right) \\
& \cdot e^{\pi i\left(2 n^{2}-2 n+1\right) \tau}=-8 \pi^{2} k\left[\left(h+4 \pi^{2} k^{2} l\right) \gamma\right. \\
& \left.+9\left(h+4 \pi^{2} k^{2} l\right) \gamma^{5}+\cdots\right],
\end{aligned}
$$

which lead to

$$
\begin{aligned}
a_{11} a_{22}-a_{12} a_{21} & =8 \pi^{2} l \gamma+o(\gamma), \\
a_{22} b_{1}-a_{12} b_{2} & =8 \pi^{2} k\left(h+4 \pi^{2} k^{2} l\right) \gamma+o(\gamma) .
\end{aligned}
$$

So, we have $\omega \rightarrow\left(h k+4 \pi^{2} k^{3} l\right) / l$ as $\operatorname{Im} \tau \rightarrow+\infty(\gamma \rightarrow 0)$. And we can write $f$ as

$$
\begin{aligned}
f= & \sum_{n=-\infty}^{+\infty} e^{\pi i n^{2} \tau+2 \pi i n \eta}=\sum_{n=-\infty}^{+\infty} e^{\pi i n^{2} \tau} e^{2 \pi i n \eta} \\
= & 1+\sum_{n=1}^{+\infty} e^{\pi i n^{2} \tau}\left(e^{2 \pi i n \eta}+e^{-2 \pi i n \eta}\right) \\
= & 1+e^{\pi i \tau}\left(e^{2 \pi i \eta}+e^{-2 \pi i \eta}\right)+e^{4 \pi i n \tau}\left(e^{4 \pi i \eta}+e^{-4 \pi i \eta}\right) \\
& +e^{9 \pi i n \tau}\left(e^{6 \pi i \eta}+e^{-6 \pi i \eta}\right)+\cdots \\
= & 1+e^{\pi i \tau} e^{2 \pi i \eta}+\left(e^{\pi i \tau} e^{-2 \pi i \eta}+e^{4 \pi i n \tau} e^{4 \pi i \eta}\right) \\
& +\left(e^{4 \pi i n \tau} e^{-4 \pi i \eta}+e^{9 \pi i n \tau} e^{6 \pi i \eta}\right)+\cdots \\
= & 1+e^{2 \pi i \eta+\pi i \tau}+e^{2 \pi i \tau}\left(e^{-2 \pi i \eta-\pi i \tau}+e^{4 \pi i \eta+2 \pi i n \tau}\right) \\
& +e^{6 \pi i n \tau}\left(e^{-4 \pi i \eta-2 \pi i \tau}+e^{6 \pi i \eta+3 \pi i \tau}\right)+\cdots
\end{aligned}
$$

It is interesting that if we set $\eta^{\prime}=2 \pi i \eta+\pi i \tau,(53)$ can be rewritten as

$$
\begin{aligned}
f= & 1+e^{\eta^{\prime}}+\gamma^{2}\left(e^{-\eta^{\prime}}+e^{2 \eta^{\prime}}\right)+\gamma^{6}\left(e^{-2 \eta^{\prime}}+e^{3 \eta^{\prime}}\right) \\
& +\cdots,
\end{aligned}
$$

From (54), we have $f \rightarrow 1+e^{\eta^{\prime}}$ as

$$
\operatorname{Im} \tau \longrightarrow+\infty(\gamma \longrightarrow 0) \text {. }
$$

Then the periodic wave solution (50) of (33) turns to the soliton

$$
\begin{aligned}
& u=2(\ln f)_{x}, \\
& f=1+e^{\eta^{\prime}}=1+e^{\pi i(2 k t+2 l y+2 h z+2 \omega t+\tau)} .
\end{aligned}
$$

\section{Conclusions and Remarks}

In this paper, we investigate a $(3+1)$-dimensional generalized shallow water wave equation (33). Its bilinear form is given by applying the relations $D_{p}$-operators and binary Bell polynomials, which has proved to be a quick and direct method. Then, we successfully get the exact periodic wave solution with the help of $D_{p}$-operators and Riemann theta function in terms of Hirota direct method. Furthermore, we obtain the corresponding soliton solutions via asymptotic analysis for their periodic wave solutions.

There are many other interesting questions on bilinear differential equations; for example, can the approach be generalized to solve trilinear equations with trilinear differential operators? How to apply the $D_{p}$-operators into the discrete equations? Besides, we will try to explore how to construct more nonlinear evolution equations with other operators simply and directly. We will continue to explore these problems in the near future.

\section{Conflict of Interests}

The authors declare that there is no conflict of interests regarding the publication of this paper.

\section{References}

[1] G.-M. Wei, Y.-T. Gao, W. Hu, and C.-Y. Zhang, "Painlevé analysis, auto-Bäcklund transformation and new analytic solutions for a generalized variable-coefficient Korteweg-de Vries (KDV) equation," The European Physical Journal B: Condensed Matter and Complex Systems, vol. 53, no. 3, pp. 343-350, 2006.

[2] Y.-Q. Yang and Y. Chen, "Pseudopotentials, lax pairs and bäcklund transformations for generalized fifth-order $\mathrm{KdV}$ equation," Communications in Theoretical Physics, vol. 55, no. 1, pp. 25-28, 2011.

[3] E. Fan, "Newbilinear Bäcklund transformation and Lax pair for the supersymmetric two-boson equation," Studies in Applied Mathematics, vol. 127, no. 3, pp. 284-301, 2011.

[4] S. Lou and X. Hu, "Broer-Kaup systems from Darboux transformation related symmetry constraints of KADomtsevPetviashvili equation," Communications in Theoretical Physics, vol. 29, no. 1, pp. 145-148, 1998.

[5] R. Hirota, "The Bäcklund and inverse scattering transform of the K-dV equation with nonuniformities," Journal of the Physical Society of Japan, vol. 46, no. 5, pp. 1681-1682, 1979.

[6] H.-H. Dong and X.-Z. Wang, "A Lie algebra containing four parameters for the generalized Dirac hierarchy," Applied Mathematics and Computation, vol. 215, no. 2, pp. 459-463, 2009.

[7] G. W. Bluman and S. Kumei, Symmetries and Differential Equations, Springer, New York, NY, USA, 1989.

[8] Y. Zhang, "A generalized Boite-Pempinelli-Tu (BPT) hierarchy and its bi-Hamiltonian structure," Physics Letters A, vol. 317, no. 3-4, pp. 280-286, 2003.

[9] W.-X. Ma, "Nonlinear continuous integrable Hamiltonian couplings," Applied Mathematics and Computation, vol. 217, no. 17, pp. 7238-7244, 2011.

[10] Y. S. Li, Soliton and Integrable System, Shanghai Scientific and Technological Education Publishing House, Shanghai, China, 1999. 
[11] W.-X. Ma, Y. Zhang, Y. Tang, and J. Tu, "Hirota bilinear equations with linear subspaces of solutions," Applied Mathematics and Computation, vol. 218, no. 13, pp. 7174-7183, 2012.

[12] A. V. Porubov and D. F. Parker, "Some general periodic solutions to coupled nonlinear Schrödinger equations," Wave Motion, vol. 29, no. 2, pp. 97-109, 1999.

[13] S. Liu, Z. Fu, S. Liu, and Q. Zhao, "Jacobi elliptic function expansion method and periodic wave solutions of nonlinear wave equations," Physics Letters A, vol. 289, no. 1-2, pp. 69-74, 2001.

[14] Z. T. Fu, S. K. Liu, S. D. Liu, and Q. Zhao, "New Jacobi elliptic function expansion and new periodic solutions of nonlinear wave equations," Physics Letters A, vol. 290, no. 1-2, pp. 72-76, 2001.

[15] H. Q. Zhang, "Extended Jacobi elliptic function expansion method and its applications," Communications in Nonlinear Science and Numerical Simulation, vol. 12, no. 5, pp. 627-635, 2007.

[16] A. Nakamura, "A direct method of calculating periodic wave solutions to nonlinear evolution equations. I. Exact twoperiodic wave solution," Journal of the Physical Society of Japan, vol. 47, no. 5, pp. 1701-1705, 1979.

[17] T.-C. Xia, B. Li, and H.-Q. Zhang, "New explicit and exact solutions for the Nizhnik-NOVikov-Vesselov equation," Applied Mathematics E-Notes, vol. 1, pp. 139-142, 2001.

[18] W. X. Ma, “Generalized bilinear differential equations," Studies in Nonlinear Sciences, vol. 2, no. 4, pp. 140-144, 2011.

[19] W.-X. Ma, "Bilinear equations and resonant solutions characterized by Bell polynomials," Reports on Mathematical Physics, vol. 72, no. 1, pp. 41-56, 2013.

[20] W.-X. Ma, "Trilinear equations, Bell polynomials, and resonant solutions," Frontiers of Mathematics in China, vol. 8, no. 5, pp. 1139-1156, 2013.

[21] S.-F. Tian and H.-Q. Zhang, "A kind of explicit Riemann theta functions periodic waves solutions for discrete soliton equations," Communications in Nonlinear Science and Numerical Simulation, vol. 16, no. 1, pp. 173-186, 2011.

[22] L. Alvarez-Gaumé, G. Moore, and C. Vafa, "Theta functions, modular invariance, and strings," Communications in Mathematical Physics, vol. 106, no. 1, pp. 1-40, 1986.

[23] Y.-H. Wang and Y. Chen, "Integrability of an extended $(2+1)$ dimensional shallow water wave equation with Bell polynomials," Chinese Physics B, vol. 22, no. 5, Article ID 050509, 2013.

[24] Y.-H. Wang and Y. Chen, "Binary Bell polynomials, bilinear approach to exact periodic wave solutions of $(2+1)$ dimensional nonlinear evolution equations," Communications in Theoretical Physics, vol. 56, no. 4, pp. 672-678, 2011. 


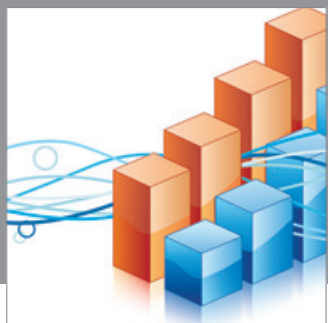

Advances in

Operations Research

mansans

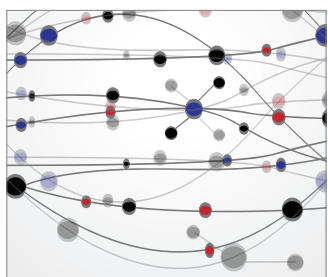

The Scientific World Journal
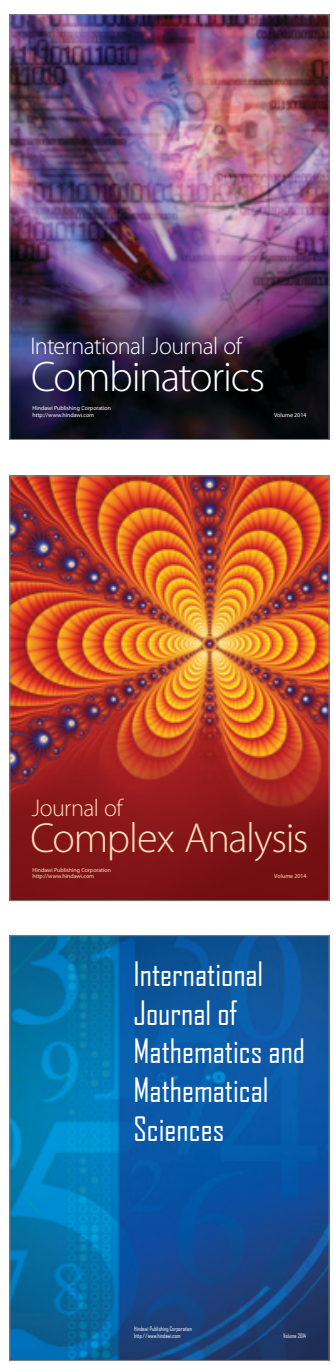
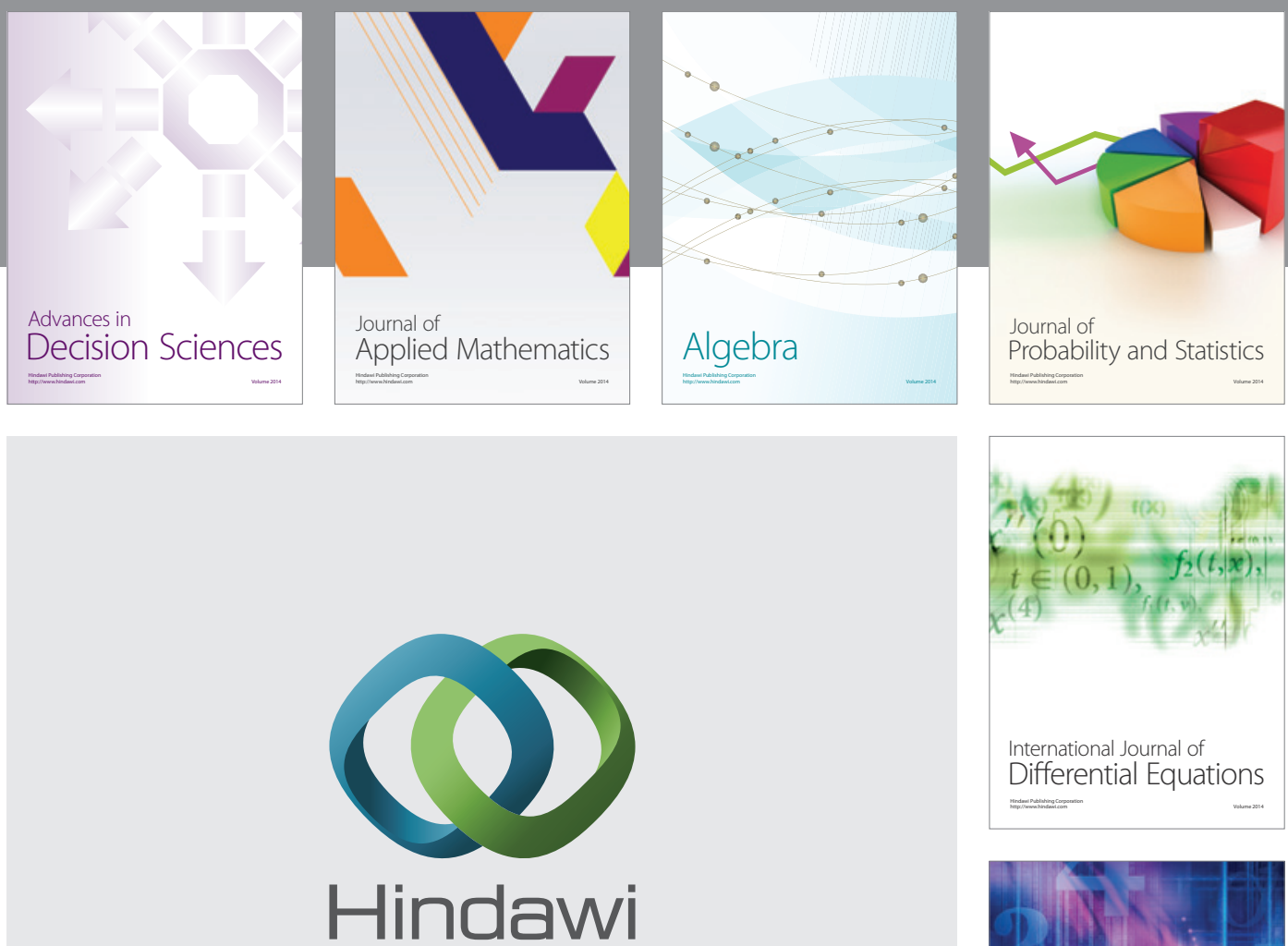

Submit your manuscripts at http://www.hindawi.com
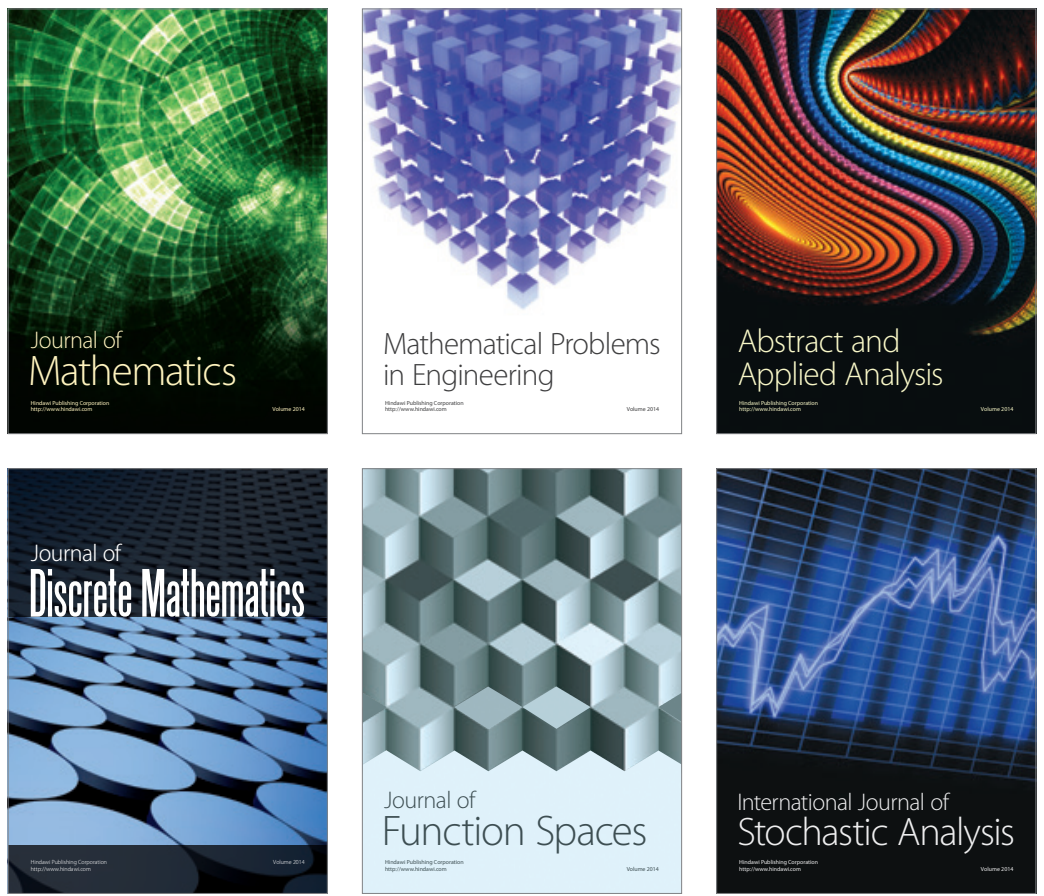

Journal of

Function Spaces

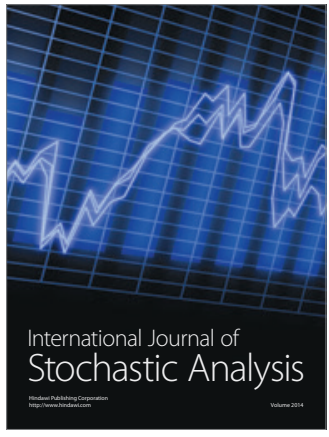

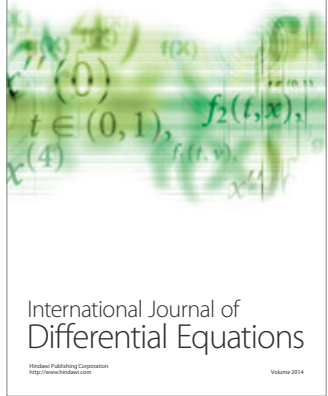
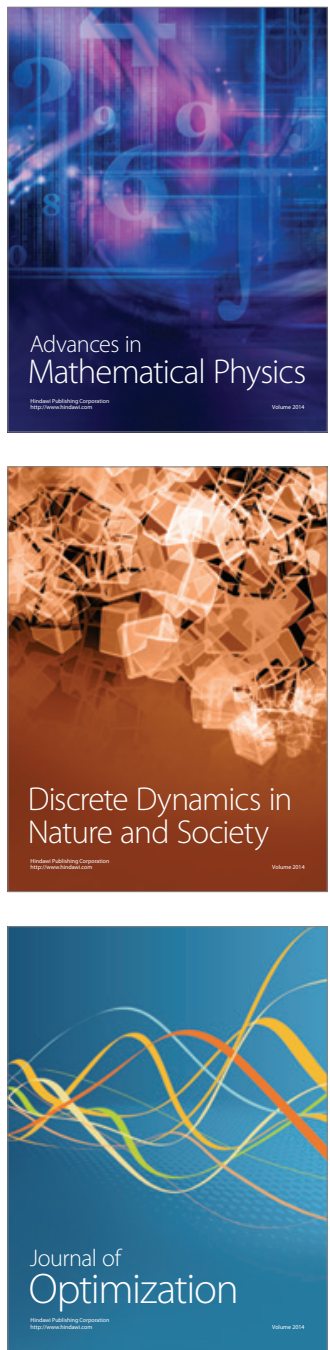